\section{References}

Ashburn, P., et al. (1973). Blood, 41, 921.

Feigin, R. D., et al. (1971). Fournal of Paediatrics, 78, 230.

Freeman, R., and King, B. (1972). Lancet, 1, 962.

Gordon, A. M., and Rowan, R. M., (1973 a). Scottish Medical Gournal, 18, 21.

Gordon, A. M., and Rowan, R. M. (1973 b). Lancet, 2, 1085.

Gordon, A. M. et al. (1973). Journal of Clinical Pathology, 26, 52.
Lancet, (1971), 2, 909.

Park, B. H. Fikrig, S. M., and Smithwick, E. M. (1968). Lancet, 2, 532. Segal, A. W., Trustey, S. F., and Levi, A. J. (1973). Lancet, 2, 879.

Siman. A. and Sasahara, A. A (1965). In Pulmonary Embolic Disease, ed. A. A. Sasahara and M. Stein. New York and London, Grune and Stratton

Vickers, P. J., and Hayes, J. P. (1973). Lancet, 2, 738.

Winchester, J. F., et al. (1973). Lancet, 2, 292.

\title{
Retention of Nitrogen, Fat, and Calories in Infants of Low Birth Weight on Conventional and High-volume Feeds
}

\author{
H. B. VALMAN \\ R. AIKENS, \\ Z. DAVID-REED, \\ J. S. GARROW
}

British Medical fournal, 1974, 3, 319-320

\section{Summary}

Two balance studies were performed on each of five infants of low birth weight. About $230 \mathrm{ml} / \mathrm{kg} /$ day of S.M.A. S26 milk was given during one study and $180 \mathrm{ml} / \mathrm{kg} /$ day during the other. The proportion of nitrogen, fat, and calories retained was similar in the two studies, suggesting that the larger weight gains on the high-volume feeds were due to growth rather than retention of water or excessive deposition of fat.

\section{Introduction}

Large gains in weight have been reported in infants of low birth weight receiving higher than conventional volumes of milk (Valman et al., 1972). Such gains could be owing to excessive salt and water retention or excessive fat deposition. We report here the results of balance studies on infants of low birth weight given both high-intake and low-intake feeds.

\section{Patients and Methods}

Five infants weighing between $1,100 \mathrm{~g}$ and $1,500 \mathrm{~g}$ at birth were studied (table $\mathrm{I}$ ). The gestational age as assessed clinically (Dubowitz et al., 1970) agreed with that calculated from the mother's last menstrual period. Two metabolic balance studies were performed on each infant, one on 230 $\mathrm{ml} / \mathrm{kg} /$ day of S.M.A. S26 milk and the other on $180 \mathrm{ml} /$ $\mathrm{kg} /$ day. Each volume was given for at least five days and collections of stool and urine were made on the final three days. Infants were weighed on scales weighing to 10 grammes.

The balance studies were performed using disposable napkins and Rayon liners as described elsewhere (Valman and Aikens, 1974). The total energy content of the excreta was determined by ballistic bomb calorimetry and the nitrogen content by Kjeldahl digestion and steam distillation. Faecal fat was estimated by the method of Van der Kamer (1958).

Northwick Park Hospital and Clinical Research Centre, Harrow, Middlesex HA1 3UJ

H. B. VALMAN, M.D., M.R.C.P., Consultant Paediatrician

R. AIKENS, S.R.N., S.C.M., Sister, Special Care Baby Unit

Z. DAVID-REED, M.R.C. Sandwich Student

J. S. GARROW, M.D., M.R.C. Scientific Staff

\begin{tabular}{|c|c|c|c|c|c|c|}
\hline \multirow{2}{*}{$\begin{array}{l}\text { Case } \\
\text { No. }\end{array}$} & \multirow{2}{*}{$\begin{array}{c}\text { Gestational } \\
\text { Age } \\
\text { (Weeks) }\end{array}$} & \multicolumn{2}{|c|}{ Birth Weight } & \multirow{2}{*}{$\begin{array}{c}\text { Balance } \\
\text { Study } \\
\text { No. }\end{array}$} & \multirow{2}{*}{$\begin{array}{l}\text { Age at } \\
\text { Start of } \\
\text { Study } \\
\text { (Days) }\end{array}$} & \multirow{2}{*}{$\begin{array}{l}\text { Weight at } \\
\text { Start of } \\
\text { Study } \\
\text { (g) }\end{array}$} \\
\hline & & (g) & Centile & & & \\
\hline 1 & 32 & 1,460 & 10\{ & 1 & $\begin{array}{l}12 \\
18\end{array}$ & $\begin{array}{l}1,480 \\
1,620\end{array}$ \\
\hline 2 & 32 & 1,160 & $<10\{$ & $\begin{array}{l}3 \\
4\end{array}$ & $\begin{array}{l}21 \\
26\end{array}$ & $\begin{array}{l}1,320 \\
1,440\end{array}$ \\
\hline 3 & 33 & 1,500 & $<10\{$ & $\begin{array}{l}5 \\
6\end{array}$ & $\begin{array}{l}26 \\
33\end{array}$ & $\begin{array}{l}1,950 \\
2,200\end{array}$ \\
\hline 4 & 28 & 1,100 & 50\{ & $\begin{array}{l}7 \\
8\end{array}$ & $\begin{array}{l}47 \\
52\end{array}$ & $\begin{array}{l}1,900 \\
2,110\end{array}$ \\
\hline 5 & 29 & 1,130 & & $\begin{array}{r}9 \\
10 \\
11\end{array}$ & $\begin{array}{l}30 \\
41 \\
47\end{array}$ & $\begin{array}{l}1,500 \\
1,960 \\
2,180\end{array}$ \\
\hline
\end{tabular}

The study was approved by the hospital ethical committee and informed consent was obtained from each mother.

\section{Results}

The percentage of the intake of nitrogen, fat, and calories which was retained was similar in the studies performed during the high and low intakes (tables II-IV). The retention of nitrogen is expressed as absolute amounts in the diagram.

\begin{tabular}{|c|c|c|c|c|c|c|}
\hline \multirow{2}{*}{$\begin{array}{l}\text { Case } \\
\text { No. }\end{array}$} & \multirow{2}{*}{$\begin{array}{c}\text { Balance } \\
\text { Study } \\
\text { No. }\end{array}$} & \multirow{2}{*}{$\begin{array}{l}\text { Approximate } \\
\text { Milk Intake } \\
\text { (ml/kg/day) }\end{array}$} & \multicolumn{4}{|c|}{ Nitrogen ( $\mathrm{g} / 3$ days) } \\
\hline & & & Intake & Stool & Urine & $\begin{array}{c}\text { Retention } \\
(\%)\end{array}$ \\
\hline 1 & $\begin{array}{l}1 \\
2\end{array}$ & $\begin{array}{l}260 \\
190\end{array}$ & $\begin{array}{l}2.918 \\
2 \cdot 371\end{array}$ & $\begin{array}{l}0.531 \\
0.350\end{array}$ & $\begin{array}{l}0.926 \\
0.893\end{array}$ & $\begin{array}{l}50 \\
48\end{array}$ \\
\hline 2 & $\begin{array}{l}3 \\
4\end{array}$ & $\begin{array}{l}260 \\
180\end{array}$ & $\begin{array}{l}2.647 \\
1.970\end{array}$ & $\begin{array}{l}0.493 \\
0.370\end{array}$ & $\begin{array}{l}0.670 \\
0.593\end{array}$ & $\begin{array}{l}56 \\
51\end{array}$ \\
\hline 3 & $\begin{array}{l}5 \\
6\end{array}$ & $\begin{array}{l}230 \\
180\end{array}$ & $\begin{array}{l}3.423 \\
3.20\end{array}$ & $\begin{array}{l}0.466 \\
0.340\end{array}$ & $\begin{array}{l}1.029 \\
0.994\end{array}$ & $\begin{array}{l}56 \\
58\end{array}$ \\
\hline 4 & $\begin{array}{l}7 \\
8\end{array}$ & $\begin{array}{l}230 \\
180\end{array}$ & $\begin{array}{l}3.360 \\
2.877\end{array}$ & $\begin{array}{l}0.517 \\
0.409\end{array}$ & $\begin{array}{l}0.842 \\
0.890\end{array}$ & $\begin{array}{l}60 \\
55\end{array}$ \\
\hline 5 & $\begin{array}{r}9 \\
10 \\
11\end{array}$ & $\begin{array}{l}200 \\
260 \\
200\end{array}$ & $\begin{array}{l}2 \cdot 351 \\
3 \cdot 955 \\
3 \cdot 285\end{array}$ & $\begin{array}{l}0.254 \\
0.261 \\
0.267\end{array}$ & $\begin{array}{l}0.724 \\
1.077 \\
0.972\end{array}$ & $\begin{array}{l}58 \\
66 \\
62\end{array}$ \\
\hline
\end{tabular}

TABLE III-Results of Fat Balance Studies in Five Infants of Low Birth Weight

\begin{tabular}{|c|c|c|c|c|}
\hline \multirow{2}{*}{$\begin{array}{l}\text { Case } \\
\text { No. }\end{array}$} & \multirow{2}{*}{$\begin{array}{c}\text { Balance } \\
\text { Study No. }\end{array}$} & \multicolumn{3}{|c|}{ Fat (g/day) } \\
\hline & & Intake & Stool & Retention (\%) \\
\hline & $\frac{1}{2}$ & $\begin{array}{l}13.8 \\
11.2\end{array}$ & $\begin{array}{l}3.28 \\
2.06\end{array}$ & $\begin{array}{l}76 \\
81\end{array}$ \\
\hline 2 & $\begin{array}{l}3 \\
4\end{array}$ & $\begin{array}{r}12.5 \\
9.3\end{array}$ & $\begin{array}{l}3.6 \\
3.5\end{array}$ & $\begin{array}{l}71 \\
62\end{array}$ \\
\hline 3 & 5 & $\begin{array}{l}16.5 \\
14.8\end{array}$ & $\begin{array}{l}2.22 \\
1.26\end{array}$ & 87 \\
\hline & 7 & $16 \cdot 2$ & 2.93 & 82 \\
\hline & 8 & $\begin{array}{l}13 \cdot 6 \\
10 \cdot 8\end{array}$ & $\begin{array}{l}1.74 \\
1.2\end{array}$ & 89 \\
\hline 5 & $\begin{array}{l}10 \\
11\end{array}$ & $\begin{array}{l}18.8 \\
15.8\end{array}$ & $\begin{array}{l}1.9 \\
0.7\end{array}$ & $\begin{array}{l}90 \\
96\end{array}$ \\
\hline
\end{tabular}




\begin{tabular}{|c|c|c|c|c|c|}
\hline \multirow{2}{*}{$\begin{array}{l}\text { Case } \\
\text { No. }\end{array}$} & \multirow{2}{*}{$\begin{array}{c}\text { Balance } \\
\text { Study } \\
\text { No. }\end{array}$} & \multicolumn{4}{|c|}{ Kilocalories/3 days } \\
\hline & & Intake & Stool & Urine & Retention (\%) \\
\hline 1 & $\frac{1}{2}$ & $\begin{array}{l}804 \\
653\end{array}$ & $\begin{array}{r}116 \\
72\end{array}$ & $\begin{array}{l}34 \\
27\end{array}$ & $\begin{array}{l}81 \\
85\end{array}$ \\
\hline 2 & $\begin{array}{l}3 \\
4\end{array}$ & $\begin{array}{l}729 \\
543\end{array}$ & $\begin{array}{r}128 \\
86\end{array}$ & $\begin{array}{l}34 \\
21\end{array}$ & $\begin{array}{l}78 \\
80\end{array}$ \\
\hline 3 & $\begin{array}{l}5 \\
6\end{array}$ & $\begin{array}{l}896 \\
800\end{array}$ & $\begin{array}{r}126 \\
66\end{array}$ & $\begin{array}{l}34 \\
32\end{array}$ & $\begin{array}{l}82 \\
88\end{array}$ \\
\hline 4 & $\begin{array}{l}7 \\
8\end{array}$ & $\begin{array}{l}880 \\
738\end{array}$ & $\begin{array}{l}168 \\
155\end{array}$ & $\begin{array}{l}48 \\
42\end{array}$ & $\begin{array}{l}76 \\
73\end{array}$ \\
\hline 5 & $\begin{array}{r}9 \\
10 \\
11\end{array}$ & $\begin{array}{l}585 \\
983 \\
854\end{array}$ & $\begin{array}{l}70 \\
88 \\
41\end{array}$ & $\begin{array}{l}42 \\
30 \\
44\end{array}$ & $\begin{array}{l}81 \\
88 \\
90\end{array}$ \\
\hline
\end{tabular}

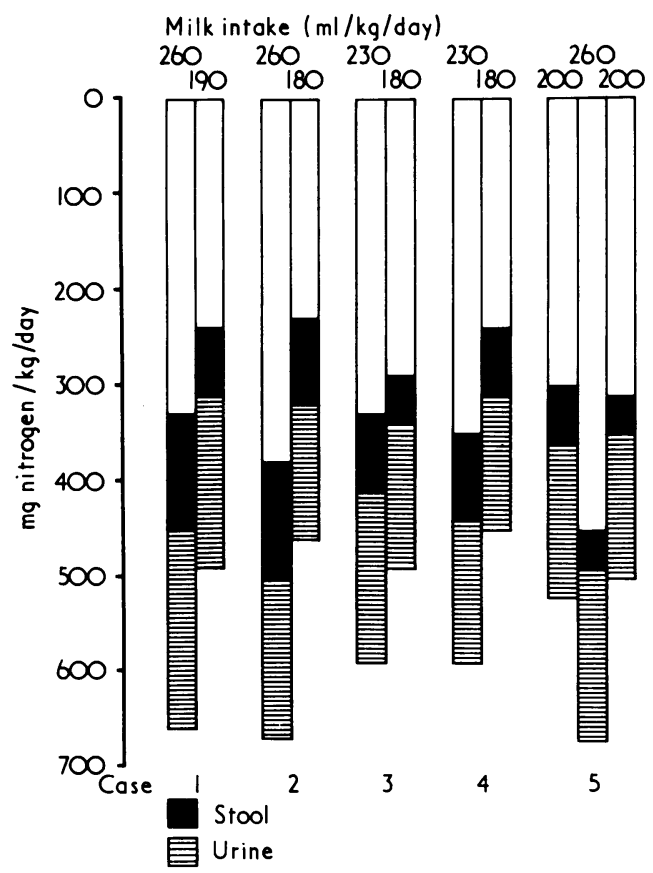

Retention of nitrogen in five infants on high-intake and low-intake feeds.

The percentage of the intake retained was similar in two low-intake studies separated by a high-intake study.

There was an enhanced weight gain during the high-intake periods compared with the low-intake periods except in cases 1 and 2 (table $\mathrm{V}$ ).

\begin{tabular}{|c|c|c|c|c|c|}
\hline \multirow[t]{2}{*}{$\begin{array}{l}\text { Case } \\
\text { No. }\end{array}$} & \multirow[t]{2}{*}{$\begin{array}{l}\text { Balance } \\
\text { Study No. }\end{array}$} & \multirow[t]{2}{*}{$\begin{array}{l}\text { Milk Intake } \\
\text { ( } \mathrm{ml} / 3 \text { days })\end{array}$} & \multirow[t]{2}{*}{$\begin{array}{l}\text { No. of Feeds } \\
/ 24 \text { hours }\end{array}$} & \multicolumn{2}{|c|}{$\begin{array}{c}\text { Weight Gain During } \\
\text { Balance }\end{array}$} \\
\hline & & & & g/day & $\mathrm{g} / \mathrm{kg} /$ day \\
\hline 1 & $\begin{array}{l}1 \\
2\end{array}$ & $\begin{array}{r}1,152 \\
936\end{array}$ & $\begin{array}{l}\text { Drip } \\
\text { Drip }\end{array}$ & $\begin{array}{l}37 \\
40\end{array}$ & $\begin{array}{l}25 \\
24\end{array}$ \\
\hline 2 & $\begin{array}{l}3 \\
4\end{array}$ & $\begin{array}{r}1,045 \\
778\end{array}$ & $\begin{array}{l}\text { Drip } \\
\text { Drip }\end{array}$ & $\begin{array}{l}20 \\
23\end{array}$ & $\begin{array}{l}15 \\
16\end{array}$ \\
\hline 3 & $\begin{array}{l}5 \\
6\end{array}$ & $\begin{array}{l}1,379 \\
1,231\end{array}$ & $\begin{array}{l}12 \\
12\end{array}$ & $\begin{array}{l}41 \\
25\end{array}$ & $\begin{array}{l}20 \\
11\end{array}$ \\
\hline 4 & $\begin{array}{l}7 \\
8\end{array}$ & $\begin{array}{l}1,354 \\
1,136\end{array}$ & $\begin{array}{r}12 \\
8\end{array}$ & $\begin{array}{l}51 \\
33\end{array}$ & $\begin{array}{l}26 \\
16\end{array}$ \\
\hline 5 & $\begin{array}{r}9 \\
10 \\
11\end{array}$ & $\begin{array}{r}900 \\
1,512 \\
1,314\end{array}$ & $\begin{array}{l}12 \\
12 \\
12\end{array}$ & $\begin{array}{l}30 \\
53 \\
40\end{array}$ & $\begin{array}{l}20 \\
27 \\
18\end{array}$ \\
\hline
\end{tabular}

\section{Discussion}

A similar percentage-and therefore a larger amount-of nitrogen, fat, and calories was retained by infants on high compared with conventional intakes. This suggests that increased intake is associated with increased growth.

The retention of nitrogen varied widely between individuals (table II) but the use of an infant as his own control allowed data to be interpreted from a small number of patients.

The fat retention was directly related to the intake (table III), and similar findings up to an intake of $9 \mathrm{~g} / \mathrm{kg} /$ day were noted by Williams et al. (1970) in full-term infants.

The third balance study in case 5 showed a similar retention of nitrogen to that in the first balance, which suggests that it is valid to compare the percentage retention on different intakes in the patients who had two balance studies.

We thank Mrs. Susan Stalley for help with nitrogen and energy determinations, Mr. George Padmore and the staff of the department of clinical chemistry for determinations of faecal fat, and Dr. George Faux of John Wyeth and Brother Ltd. for supplying S.M.A. S26 and some financial support.

\section{References}

Dubowitz, L. M. S., Dubowitz, V., and Goldberg, C. (1970). Fournal of Pediatrics, 77,1 .

Valman, H. B., Heath, C. D., and Brown, R. J. K. (1972). British Medical fournal, 3, 547 .

Valman, H. B., and Aikens, R. (1974). To be published.

Van der Kamer, J. H. (1958). In Standard Methods of Clinical Chemistry, ed. D. Seligson, Vol. 2, p. 64. New York, Academic Press.

Williams, M. L., et al. (1970). American fournal of Clinical Nutrition, 23, 1322 . 